\title{
Über einige Fehlerquellen bei der gasometrischen Nitrat- und Nitritbestimmung nach Schlösing bzw. Piccini.
}

\author{
Von \\ Otto Ruff und Ewald Gersten.
}

Die gasometrische Bestimmung der Salpeter- und salpetrigen Säure in Nitraten und Nitriten nach Schlössra ${ }^{2}$ bzw. PiccrNi ${ }^{2}$ wird noch immer so häufig angewandt, dals einige Schwierigkeiten, denen wir bei ihrem Gebrauch begegneten und einige Versuche, ihre Ursachen $\mathrm{zu}$ erkennen und soweit möglich zu beseitigen, auch allgemeineres Interesse finden dürften.

Enthalten die zu analysierenden Nitrat- oder Nitritlösungen Arsenite oder Sulfide, so wechselt die Ausbeute an Stickoxyd mit den Versuchsbedingungen und ist stets zu klein.

Durch die im nachstehenden beschriebenen Versuche konnten wir feststellen, dals die Stickoxydrerluste durch die besondere reduzierende Wirkung der arsenigen Säure auf die Salpetersäure und diejenige des Schwefelwasserstoffs auf die Salpeter- und salpetrige Säure in saurer Lösung bedingt waren. Wenn auch bereits bekannt war, dals durch Zinnchlorür und Salzsäure Salpetersäure bis zu Ammoniak reduziert werden kann und dals Stickoxyd mit Chromchlorür und Salzsäure eine überaus beständige Chromonitrosoverhindung bildet, ${ }^{3}$ dal's also die Gegenwart so stark reduzierender Stoffe die Stickoxydentwickelung zweifellos beeinträchtigen mulste,

${ }^{1}$ Schlösing, Joum, prakt. Chem. 62, 142.

Prcorir, Ber. 1881, 1168 und Pellet, Chem. Centrbl. 1900 II, 1009.

${ }^{3}$ Kocht man Nitratlösungen in Gegenwart von Chromchlorür mit Ferriehlorid und Salzsäure, so bleibt der gröbere Teil des Stickoxyds in der Lösung, wohl in Form einer Chromonitrosoverbindung gebunden; Ammoniak liefs sich . in solcher Lösung nicht nachweisen. 
so war eine solche Wirkung von der verbältnismäfsig schwachen arsenigen Säure und dem Schwefelwasserstoff a priori doch kaum zu erwarten, um so weniger als die gasometrische Methode sich gerade dann bei der Bestimmung von Nitraten und Nitriten bewährt hatte, wenn diese, wie z. B. in Düngestoffen, mit organischer Substanz vermischt waren.

\section{Einflufs der arsenigen Säure.}

Bei der Bestimmung von Nitraten, welche die Verwendung einer stark sauren Eisenchlorürlösung nötig macht, veranlafst die Gegenwart von Arsenit in der zu analysierenden Lösung um so gröfsere Verluste an Stickoxyd, je grölser die Menge der in der Lösung enthaltenen arsenigen Säure und die Salzsäurekonzentration der zu erhitzenden Lösungen ist (Versuchsreibe I).

Die Bestimmungen wurden in dem von Tremann und Schulze ${ }^{1}$ modifizierten und von L. L. DE KoNINCK ${ }^{2}$ verbesserten Apparat ausgeführt, in dessen Gasableitungsrohr wir ein T-Stück einfügten, um das Luftleerkochen der Apparatur rascher und bequemer erreichen zu können. Die zu analysierende Kaliumnitratlösung, immer $50 \mathrm{ccm}$, wurde (eventl. nach Zusatz von Arsenitlösung) erst ausgekocht, bis auf $25 \mathrm{ccm}$, dann wurden $20 \mathrm{ccm}$ gesättigte Ferrochloridlösung zugleich mit soviel Wasser und Salzsäure (alles zuror ausgekocht) in den Apparat eingelassen, dafs nach Neutralisation des Alkalis der Arsenitlösung die in der Tabelle angegebene Konzentration an freier Salzsäure noch übrig blieb und dals das Gesamtvolum der Lösung dann $70 \mathrm{ccm}$ betrug. Das entwickelte $\mathrm{Gas}(\mathrm{I}, 1)$ wurde in einem Azotometer über Natronlauge aufgefangen. Es enthielt, wie sich durch Absorption des Gases in alkalischer Natriumsulfitlösung feststellen liels, ${ }^{3}$ neben Stickoxyd (I, 2) auch noch Stickstoff (I, 3), und zwar in gröfserem Betrag als dies bei arsenitfreien Lösungen für gewöhnlich der Fall zu sein pflegte (vgl. I, a mit I,b bis e). Das Stickstoffvolum betrug durchschnittlich $0.36 \mathrm{ccm}$, während IлIEснті und RITTER einen unabsorbierbaren Rest von durchscbnittlich unter $0.1 \mathrm{ccm}$ beobachtet hatten. Bei Berücksichtigung des Mehrbetrages an Stickstoff, über $0.36 \mathrm{ccm}$ hinaus, der auf Stickoxyd umgerechnet ${ }^{1}$

1 TremanN und Scholze, Zeitschr. analyt, Chem. 9 (1870), 401. - Ber. deutsch. chem. Ges. 6 (1875), 1041.

2 L. L. DE Konisck, Zeitschr. analyt. Chem. 33, 1894.

3 Divers., Chem. Soo. Proe. 198 (1898/99), 22.

11 Vol. $\mathrm{N}_{2}$ entsprechend $2 \mathrm{Vol} . \mathrm{NO}_{2}$. 
$(I, 4)$ und dem Stickoxydvolum zugezählt wurde, wurden die Stickoxydverluste $(I, 5)$ zwar etwas verringert, aber doch nicht gänzlich beseitigt. Die Verluste waren dadurch bedingt, dals nach Zugabe der Salzsäure unter der Wirkung der arsenigen Süure die Salpetersäure teilweise zu salpetriger Säure reduziert wurde, worauf dann diese letztere zum Teil zu NO und Salpetersäure weiter zerfiel, zum Teil aber auch mit den Wasserdämpfen als solche fortgeführt und damit der direkten Bestimmung als Stickoxyd entzogen wurde. Die verlorene salpetrige Säure konnten wir in der Natronlauge des zum Auffangen des Stickoxyds bestimmten Azotometers quantitativ wiederfinden, als sie ein gedampft und mit Ferrochlorid und Salzsäure erneut destilliert wurde; aber merkwürdigerweise erhielt man sie auch aus dem Azotometerinhalt wieder zum Teil als Stickstoff (I, d). Die Verluste an Stickoxyd wurden um so gröfser, je grölser die Arsenigsäure- und je kleiner die Salzsäurekonzentration in der zu destillierenden Flüssigkeit war (vgl. Versuch I, a, b mit c und $c$ mit d).

Versuchsreibe I (0.1012 $\left.\mathrm{g}^{\mathrm{KNO}_{3}}\right)$.

\begin{tabular}{|c|c|c|c|c|c|c|c|c|c|c|c|}
\hline $\begin{array}{c}\text { Angewandte } \\
\text { Iösung }\end{array}$ & 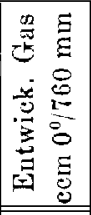 & 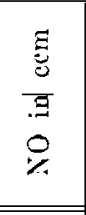 & 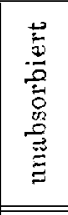 & 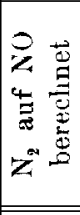 & 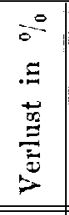 & 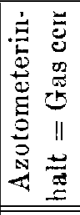 & $a^{2}$ & 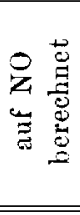 & 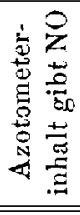 & 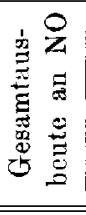 & $\begin{array}{l}0 \\
0 \\
0 \\
0 \\
z\end{array}$ \\
\hline  & 21.77 & 21.41 & 0.36 & - & - & - & - & - & - & 21.41 & - \\
\hline $\begin{array}{l}\text { b) Dieselbe Lsg. } \\
\text { mit } 0.1 \mathrm{~g} \mathrm{As} \mathrm{O}_{3} \\
2 n \mathrm{HCl}^{2}\end{array}$ & 21.18 & 20.82 & 0.36 & - & 2.71 & 0.45 & 0.12 & 0.26 & 0.59 & 21.41 & 100 \\
\hline $\begin{array}{c}\text { c) Dieselbe Lsg. } \\
\text { mit } 0.5 \mathrm{~g} \mathrm{As}_{2} \mathrm{O}_{3} \\
2 n \mathrm{HCl}\end{array}$ & 20.84 & 20.60 & 0.47 & 0.22 & 3.79 & 0.50 & 0.17 & 0.36 & 0.69 & 21.51 & 100.51 \\
\hline $\begin{array}{l}\text { d) Dieselbe Lag. } \\
\text { mit } 1.0 \mathrm{Hs}_{3} \mathrm{O}_{3} \\
2 n \mathrm{HCl}\end{array}$ & 20.88 & 20.71 & 0.52 & 0.32 & 3.27 & 0.45 & 0.15 & 0.32 & 0.62 & 21.65 & 101.12 \\
\hline $\begin{array}{l}\text { e) Dieselbe L Lsg. } \\
\text { mit } 0.1 \mathrm{~g} \mathrm{As}_{2} \mathrm{O}_{3} \\
4 n \mathrm{HCl}\end{array}$ & 21.44 & 21.13 & 0.40 & 0.08 & 1.31 & 0.21 & 0.04 & 0.08 & 0.25 & 21.46 & 100.23 \\
\hline $\begin{array}{l}\text { f) Diegelbe Isg.). } \\
\text { mit } 1.0 \mathrm{~g} \mathrm{As} \mathrm{O}_{3} \\
\text { u. } 4 \mathrm{nCl}\end{array}$ & 21.37 & 21.08 & 0.40 & 0.08 & 1.54 & 0.20 & 0.05 & 0.11 & 0.26 & 21.42 & 100.05 \\
\hline
\end{tabular}

Dafs der aufgefangene Stickstoff in allen Fällen kein Stickoxydul enthielt, stellten wir noch besonders fest, indem wir den 
nach der Absorption des Stickoxyds uns bleibenden Stickstoff mit Wasserstoff mischten und in einer Quecksilberexplosionspipette längere Zeit dem Induktionsfunken aussetzten. (Der Stickstoff verdankt jedenfalls einer besonderen neben der Stickoxydbildung hergehenden Reaktion seine Entstehung; er scheint in um so grölserer Menge aufzutreten, je grölsere Salzsäurekonzentrationen bei der Reduktion der Salpetersäure durch Ferrochlorid Verwendung finden; doch sind daneben für seine Bildung sicherlich auch noch andere uns unbekannte Umstände von Bedeutung.)

Dals unsere Erklärung von der Wirkung der arsenigen Säure richtig ist, mag auch noch die folgende Versuchsreihe zeigen, in der die Wirkung von Arsenigsäure allein (also ohne Ferrochlorid) auf Salpetersäure unter den Versuchsbedingungen der ScHsösrivgschen Methode studiert wurde.

$\mathrm{Zu}$ der ausgekochten Nitrat-Arsenitlösung wurde Salzsäure zugegeben und gekocht, bis die bald recht langsam werdende Gasentwickelung beendet war; es blieb bei ausreichenden Arsenigsäuremengen im Kolben keinerlei Stickstoffrerbindung zurück. Das im Azotometer aufgefangene Gas wurde wieder wie oben analysiert, in Versuch c) ebenso auch die Azotometerflüssigkeit. Es zeigt sich,

$$
\text { Versuchsreihe II }\left(0.1011 \mathrm{~g} \mathrm{KNO}_{3}\right) \text {. }
$$

Gesamtrolum $50 \mathrm{ccm}$.

\begin{tabular}{|c|c|c|c|c|c|}
\hline & $\begin{array}{c}1 \\
\text { Zusammensetzung } \\
\text { der Lösung }\end{array}$ & $\begin{array}{c}2 \\
\text { Gas } \\
\mathrm{ccm}\end{array}$ & $\begin{array}{c}3 \\
\text { biervon } \mathrm{N}_{2} \\
\text { cem }\end{array}$ & $\begin{array}{c}4 \\
\text { Verlust } \\
\%\end{array}$ & $\begin{array}{c}5 \\
\text { Bemerkungen }\end{array}$ \\
\hline & $\left.\begin{array}{c}20 \operatorname{cem} \mathrm{FeCl}_{2}-\mathrm{Lsg} . \\
\mathrm{H}_{2} \mathrm{O}+\mathrm{HCl} \text { bis } \\
3_{11}-\text { norm. }\end{array}\right\}$ & $\begin{array}{l}22.01 \\
(22.4 \\
\text { theor. })\end{array}$ & 0.26 & 0 & Normalbestimmung \\
\hline a) & $\begin{array}{c}0.25 \mathrm{~g} \mathrm{As}_{2} \mathrm{O}_{3} \\
\mathrm{H}_{2} \mathrm{O}+\mathrm{HCl} \text { bis } \\
4 \text {. norm. }\end{array}$ & 10.83 & 0.30 & 50 & \\
\hline b) & $\begin{array}{c}0.5 \mathrm{~g} \mathrm{As}_{2} \mathrm{O}_{3} \\
\mathrm{H}_{2} \mathrm{O}+\mathrm{HCl} \text { bis } \\
4 / 1 \text {-norm. }\end{array}$ & 14.44 & 0.27 & 33.9 & $\begin{array}{l}\text { Dalzsaure- } \\
\text { konzentration } \\
\text { konstant }\end{array}$ \\
\hline c) & $\begin{array}{l}1.00 \mathrm{~g} \mathrm{Aa_{2 } \mathrm { O } _ { 3 }} \\
\text { sonst wie vor }\end{array}$ & 14.38 & 0.32 & 33.9 & \\
\hline d) & $\begin{array}{c}0.5 \mathrm{~g} \mathrm{As} \mathrm{As}_{3} \\
\mathrm{H}_{2} \mathrm{O}+\mathrm{HCl} \text { bis } \\
1,75 / 2 \text {-norm. }\end{array}$ & 3.24 & 0.25 & 84.3 & $\begin{array}{l}\text { Salzsïure- } \\
\text { konzentration } \\
\text { kleiner }\end{array}$ \\
\hline e) & 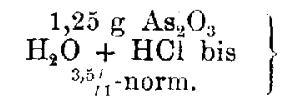 & 12.80 & 0.3 & 41.2 & $\begin{array}{l}\text { Azotometerinhalt } \\
\text { analysiert s. u. }\end{array}$ \\
\hline
\end{tabular}


dafs bei Gegenwart hinreichend grofser Arsenigsäuremengen alle Salpetersäure reduziert wird und quantitativ zum Teil als Stickoxyd, zum Teil als salpetrige Säure in das Azotometer übergeht (II, e); hierbei ist für die Menge der überdestillierenden salpetrigen Säure die Arsenigsäurekonzentration nur so lange von Bedeutung, als es sich um kleinere Mengen handelt (vgl. II, a mit $b$ und $c$ ), von um so gröfserer dagegen die Salzsäurekonzentration (vgl. II, b mit d). Der Gasverlust wurde um so gröfser, je kleiner die Salzsäurekonzentration war.

Der Azotometerinhalt von diesem Versuche ergab bei der Destillation mit Ferrochlorid und Salzsäure $5.86 \mathrm{ccm}$ Gas, enthaltend $2.97 \mathrm{~cm} \mathrm{~N}_{2}$ und $3.89 \mathrm{ccm} \mathrm{NO}$, so dals die Gesamtmenge entwickelten Gases $15.39 \mathrm{ccm}$ NO und $3.27 \mathrm{~N}_{2}=6.54 \mathrm{ccm}$ NO= $\frac{21.93 \cdot 100}{22.27}=98.3 \%$ der Theorie beträgt.

In neutraler oder alkalischer Lösung wird eine Reduktion des Nitrats durch das Arsenit zu Stickoxyd oder Nitrit merkwürdigerweise nicht herbeigeführt, obwohl die reduzierende Kraft der arsenigen Säure in saurer Lösung geringer ist als in alkalischer. ${ }^{1}$ Sie geht vielmehr erst in saurer Lösung vor sich. Wir konnten dies beweisen, indem wir nach längerem Kochen von Nitratlösungen mit Arsenitlösungen das Arsenit und das möglicherweise gebildete Arseniat durch eine Mischung von Kalkwasser und Calciumchloridlösung fällten und das Filtrat von Calciumarsenit und Arseniat qualitativ und quantitativ auf salpetrige Säure prüften. Das erstere geschah mit Hilfe von Diphenylaminschwefelsäure und ergab überhaupt keine Reaktion. Das zweite durch Oxydation des Filtrats mit Permanganat in saurer Lösung. Hierbei verbrauchten Filtrate von reinem Calciumarsenit fast genau dieselbe Menge Permanganat wie Filtrate von Calciumarsenit, die aus Nitratlösungen in der oben geschilderten Weise erhalten worden waren.

Für die Bestimmung von Nitriten diente uns reines Silbernitrit als Ausgangsmaterial, das nach der Vorschrift von Treadwel ${ }^{2}$ mit Chlorkalium umgesetzt wurde. Wir überzeugten uns zunächst, dals entsprechend den Angaben von Piccini und Peluet (l. c.) aus Nitritlösungen beim Kochen mit Eisenchlorürlösung ohne Salzsäurezusatz die berechneten Mengen Stickoxyd entwickelt wurden, dafs

1 Anegg, Handb. d. anorg. Chem. III 3, S. 535.

Treajweld, Lehrb. d. analyt. Chem. 1907 II, S. 256. 
dagegen bei Verwendung freier Salzsäure zu geringe Mengen erhalten wurden. (Versuchsreihe III, a und b.)

Wir fanden auch in diesem Falle alles fehlende Stickoxyd als salpetrige Säure im Azotometer wieder. Das aus dem Azotometerinhalt entwickelte Stickoxyd erwies sich aber im Gegensatz zu den früheren Versuchen als stickstofffrei (III, b).

Fügt man der Nitritlösung Arsenitlösung hinzu (III, c), so erhält man ohne Salzsäurezusatz wiederum die theoretischen Werte; die Gegenwart ron Arsenit schadet in diesem Falle also gar nichts; freie Säure macht die Bestimmung natürlich fehlerhaft (III, d); doch lassen sich die Verluste dadurch erheblich einschränken, dafs man auf dem Siedekolben einen Rückflufskühler anbringt, der die Salpetrigsäure zurückhält (III, e), so dafs sie vom Eisensalz allmählich fast vollständig zu Stickoxyd reduziert werden kann. Die Ferrochloridlösung läfst sich ohne weiteres durch Ferrosulfatlösung ersetzen (III, f), doch darf auch in solchem Fall neben dem Ferrosulfat freie Schwefelsäure nicht Verwendung finden.

Versuchsreihe III (50 ccm Nitritlösung entspr. $21.85 \mathrm{ccm} \mathrm{NO}$ ). Gesamtvolum $70 \mathrm{ccm}$.

\begin{tabular}{|c|c|c|c|c|c|}
\hline & $\begin{array}{l}\text { Zusammensetzung } \\
\text { der Lösung }\end{array}$ & $\begin{array}{l}\text { Gas } \\
\mathrm{ccm}\end{array}$ & $\begin{array}{c}\text { biervon } \mathrm{N}_{2} \\
\operatorname{ccm}\end{array}$ & $\begin{array}{c}\text { Verlust } \\
\%\end{array}$ & Bemerkungen \\
\hline a) & $20 \mathrm{~cm} \mathrm{FeCl}{ }_{2}$-Lsg. & 21.78 & 0.07 & 0 & Normalbestimmung \\
\hline b) & $\begin{array}{c}\text { Dasselbe in 1.8-norm. } \\
\mathrm{HCl}\end{array}$ & 15.95 & 0.49 & 24.8 & \begin{tabular}{||c} 
Der Azotometer- \\
inhalt ergab noch \\
$5.56 \mathrm{ccm} \mathrm{NO}=25.4^{0} \%$
\end{tabular} \\
\hline c) & 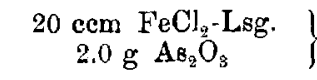 & 21.7 & 0.1 & 0.3 & \\
\hline d) & $\begin{array}{c}\text { Dasselbe in 2.5-norm. } \\
\mathrm{HCl}\end{array}$ & 17.75 & 0.33 & 17.2 & \begin{tabular}{||c} 
Der Azotometer- \\
inhalt ergab noch \\
3.61 cem $\mathrm{NO}=16.7^{\circ} \%$
\end{tabular} \\
\hline e) & $\begin{array}{c}\text { Dasselbe, jedoch mit } \\
\text { Rückflufskühler }\end{array}$ & 19.95 & 0.31 & 7.3 & \begin{tabular}{|l} 
Der Azotometer- \\
inhalt ergab noch \\
$1.19 \mathrm{ccm} \mathrm{NO}=5.4^{\circ} \%$
\end{tabular} \\
\hline f) & $20 \mathrm{ccm} \mathrm{FeSO}{ }_{4}$-Lsg. & 21.7 & 0.09 & 0.3 & \\
\hline g) & $\begin{array}{c}\text { Dasselbe in } 0.6 \text { norm. } \\
\mathrm{H}_{2} \mathrm{SO}_{4}\end{array}$ & 18.32 & 0.1 & 15.7 & \begin{tabular}{|l} 
Der Azotometerin \\
balt ergab noch 2.81 \\
cem $\mathrm{NO}=12.85^{\circ}$
\end{tabular} \\
\hline
\end{tabular}

Es ist also auch diese Versuchsreihe ein Beleg dafür, dals die bei der Bestimmung von Nitraten durch die Gegenwart vor Arsenigsäure veranlafsten Störungen lediglich von einer Reduktion der Salpetersäure zu salpetriger Säure herrühren. 


\section{Einflufs des Schwefelwasserstoffs.}

Nitratbestimmungen. Die Versuche wurden in der Weise angestellt, dafs zur luftleer gekochten Nitratlösung ausgekochte Natriumsulfidlösung und Eisenchlorürlösung hinzugegeben wurde und dann Salzsäure im Überschufs. Hierbei löste sich unter Schwefelwasserstoffentwickelung das Ferrosulfid und es bildete sich teils schon in der Kälte, teils erst beim Kochen Stickoxyd, das in alkalischer Natriumsulfitlösung vollständig löslich war, also ausschliefslich aus Stickoxyd bestand. Der Schwefelwasserstoff wurde ron der alkalischen Azotometerflüssigkeit aufgenommen. Die erhaltenen Stickoxydmengen waren viel zu klein; Salpetrigsäure liefs sich in der Azotometerflüssigkeit nicht nachweisen, dafür faud sich in der Reaktionsflüssigkeit Ammoniak, und zwar genau in derjenigen Menge, um die zu wenig Stickoxyd entwickelt worden war. (Versuchsreibe $I V$, $a$ und $b$.)

Nitritbestimmungen. Versuche mit Nitritlösungen zeigten das gleiche Ergebnis. Es wurden bei diesen nach dem Zusatz ron Eisenchlorür zu der natriumsulfidhaltigen Nitritlösung nur soviel Salzsäure verwendet, dals gerade alles Schwefeleisen gelöst war. ( $\nabla$ ersuchsreihe IV, $c$ und d.)

Versuchsreihe IV.

\begin{tabular}{|c|c|c|c|c|}
\hline & Angewandte Jösung & $\begin{array}{c}\text { Entwickeltes } \\
\text { Gas }\end{array}$ & $\begin{array}{c}\text { Gebildetes Am- } \\
\text { moniak in cem NO }\end{array}$ & $\begin{array}{l}\text { Gesamt- } \\
\text { ausbeute }\end{array}$ \\
\hline a) & $\begin{array}{c}0.1001 \mathrm{~g} \mathrm{KNO} \mathrm{KN}_{3} 20 \mathrm{ecm} \mathrm{FeCl}_{2}- \\
\text { Lag. } 30 \mathrm{ecm} \mathrm{HCl}\end{array}$ & 20.95 & - & 100 \\
\hline b) & Dasselbe mit $10 \mathrm{ccm} \mathrm{Na}_{2} \mathrm{~S}$-Lsg. & 16.70 & 4.40 & 100.5 \\
\hline c) & $\begin{array}{c}0.0805 \mathrm{~g} \mathrm{KNO} \text { in } 50 \mathrm{ccm} \\
20 \mathrm{ccm} \mathrm{FeUl}-I_{2} \mathrm{sg} .\end{array}$ & 21.66 & - & 100 \\
\hline d) & 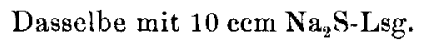 & 15.20 & 6.24 & $99 \%$ \\
\hline
\end{tabular}

Es ergibt sich also aus diesen Versuchen, dal's die gasometrische Bestimmung von Nitraten nach Schlösing in Gegenwart ron arseniger Säure oder von Schwefelwasserstoff zu niedrige Werte ergibt - im Falle der arsenigen Säure dadurch, dals ein Teil der Salpetersäure zu Salpetrigsäure reduziert und als solche in die Azotometerflüssigkeit übergeführt wird, im Falle des Schwefelwasserstoffs dadurch, dafs neben Stickoxyd auch noch Z. anorg. Chem, Bd. $i 1$. 


\section{$-426$}

Ammoniak gebildet wird, das in der sauren Reaktionsflüssigkeit zurückbleibt. Die Bestimmung von Nitriten nach Procini wird durch die Gegenwart von arseniger Säure nichtbeeinträchtigt, wenn man in neutraler Lösung arbeitet; sie wird aber durch die Gegenwart von Sulfiden unmöglich gemacht, indem Schwefelwasserstoff die salpetrige Säure zum Teil zu Ammoniak reduziert.

Danzig, Anorgan. u. elektrochem. Laboratorium der Kgl. Techn. Hochschule.

Bei der Redaktion eingegangen am 25. Juni 1911. 